\title{
Editorial
}

\section{Beamforming Techniques for Wireless MIMO Relay Networks}

\author{
Athanasios G. Kanatas, ${ }^{1}$ Demosthenes Vouyioukas, ${ }^{2}$ Gan Zheng, ${ }^{3}$ and Laurent Clavier ${ }^{4}$ \\ ${ }^{1}$ Department of Digital Systems, School of ICT, University of Piraeus, 18534 Piraeus, Greece \\ ${ }^{2}$ Department of Information and Communication Systems Engineering, University of the Aegean, \\ 83200 Karlovassi, Samos Island, Greece \\ ${ }^{3}$ School of Computer Science and Electronic Engineering, University of Essex, Wivenhoe Park, Colchester CO4 3SQ, UK \\ ${ }^{4}$ IEMN, UMR CNRS 8520 and IRCICA, USR CNRS 3380 and Telecom Lille, Institut Mines Telecom, Lille, France \\ Correspondence should be addressed to Athanasios G. Kanatas; kanatas@unipi.gr
}

Received 12 December 2013; Accepted 12 December 2013; Published 22 July 2014

Copyright (C) 2014 Athanasios G. Kanatas et al. This is an open access article distributed under the Creative Commons Attribution License, which permits unrestricted use, distribution, and reproduction in any medium, provided the original work is properly cited.

The continuous increase in mobile data traffic creates the need for radical innovations in the mobile broadband system design. The demand for high-speed and interference-free transmission and reception is inevitable and one sine qua non condition is the efficient spatial reuse. However, increasing traffic within a fixed limited bandwidth creates more interference in the system and degrades the signal quality.

Multiple-input multiple-output (MIMO) techniques offer many benefits in practical wireless systems including capacity and spectral efficiency increment, fading mitigation, and improved resistance to interference. Beamforming is a multiantenna technique that significantly reduces interference and improves system capacity.

An emerging research trend considers relays in terrestrial and satellite networks as the most promising proposal for achieving significant performance gains. Enhanced reliability and extended cell coverage are two of the advantages the relay networks offer. Relay networks encompass amplify-andforward (AF) and decode-and-forward (DF) protocols, fulland half-duplex operation, experience fading and interference, and incorporate power constraints.

Although beamforming techniques at the source and/or the destination in a relay network have been examined, their use in MIMO relay networks has been recently proposed and is expected to overcome crucial obstacles in terms of capacity and interference. It is thus expected to contribute to the performance of the network, while combining various attributes of networks' design parameters.
The objective of this special issue is to provide the technology development and an in-depth description of the stateof-the-art on the area of beamforming and MIMO relay networks. We received 11 paper submissions and finally accepted 5 according to the reviewers' comments and associate editors' suggestions. The accepted papers cover the topics of radiation antenna pattern design for LTE base stations and relays, integrating MIMO systems and MISO beamforming, along with opportunistic MIMO beamforming power management issues for effective Quality of Service (QoS) fulfillment. Moreover, the special issue includes a survey article that presents the state-of-the-art and the major trends of the applied beamforming techniques on MIMO relay networks.

Specifically, two papers are concentrated on antenna design; one paper proposes effective radiation pattern (ERP) method that is incorporated into a multicell bad urban $4 \mathrm{G}$ LTE operational scenario. It employs beamforming for both the base stations (BSs) and the relay nodes (RNs) in order to demonstrate the effects of the radio channel spatial characteristics on system level measures (SIR, capacity) as well as the validity of the proposed method. The other one is focused on a novel MIMO transmitter built on parasitic antenna arrays that shapes directly the radiation pattern with the aid of analog tunable loads attached to the parasitics, which is able to provide complex loading values even with negative real part and thus high order modulation schemes.

Two papers deal with beamforming methods and their performance with distinctive approaches; one paper studies 
the optimum placement of the antenna elements in a given area that can maximize the ergodic capacity of a multi-input single-output (MISO) beamforming system. It is found that it is highly related to the power azimuth spectrum (PAS) distribution and power elevation spectrum (PES). The next paper explores a transmit power management mechanism where multiuser MIMO opportunistic beamforming with adaptive modulation strategy is performed by the system, satisfying the Quality of Service (QoS) for the scheduled user.

Finally, a comprehensive survey, mainly focused on the performance of the adopted beamforming techniques on MIMO relay networks, is presented. The main objective is to provide an account of recent research activity under various network performance challenges. The paper focuses on recently developed procedures for interference modeling and mitigation, beamforming channel modeling, channel estimation and feedback, complexity and power consumption, adaptive beamforming for multiuser relaying, degrees of freedom, diversity issues, and spectral efficiency in cooperative and opportunistic systems.

As long as different beamforming methods and MIMO network topologies and techniques have been considered, pertaining the challenges of beamforming implementation in MIMO relay networks, we sincerely hope that this special issue can further help the readers to understand beamforming in the context of wireless MIMO and relay systems and enhance their study for its potential application on the forthcoming $5 \mathrm{G}$ system.

\section{Acknowledgments}

We hope that this special issue will attract the attention of other researchers in this area. We would like to express our appreciation to all the authors and reviewers for their great support that made this issue possible.

Athanasios G. Kanatas

Demosthenes Vouyioukas

Gan Zheng

Laurent Clavier 

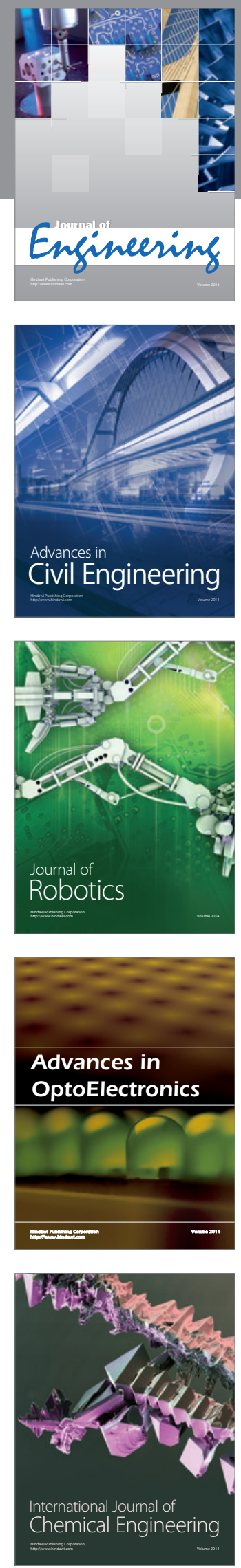

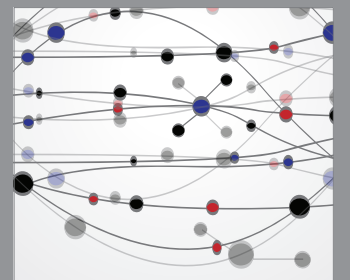

The Scientific World Journal
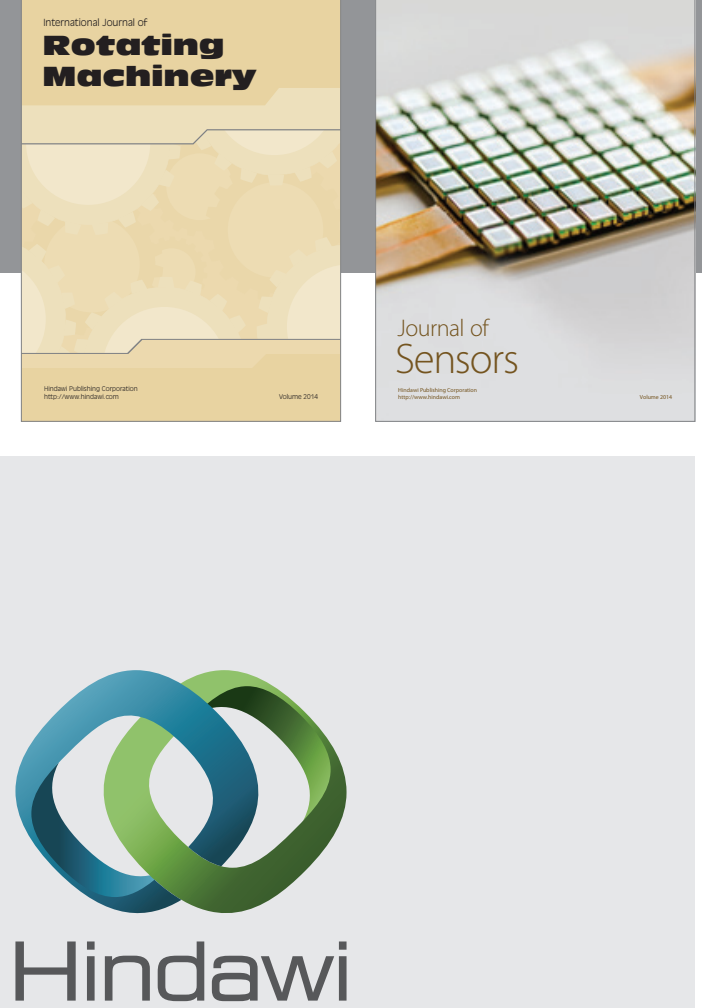

Submit your manuscripts at http://www.hindawi.com
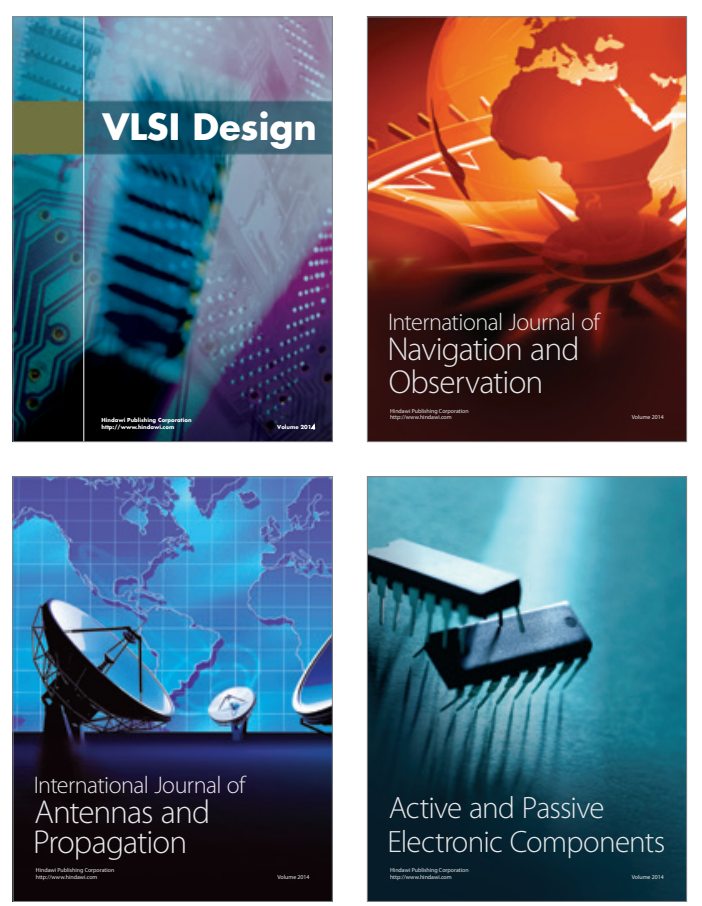
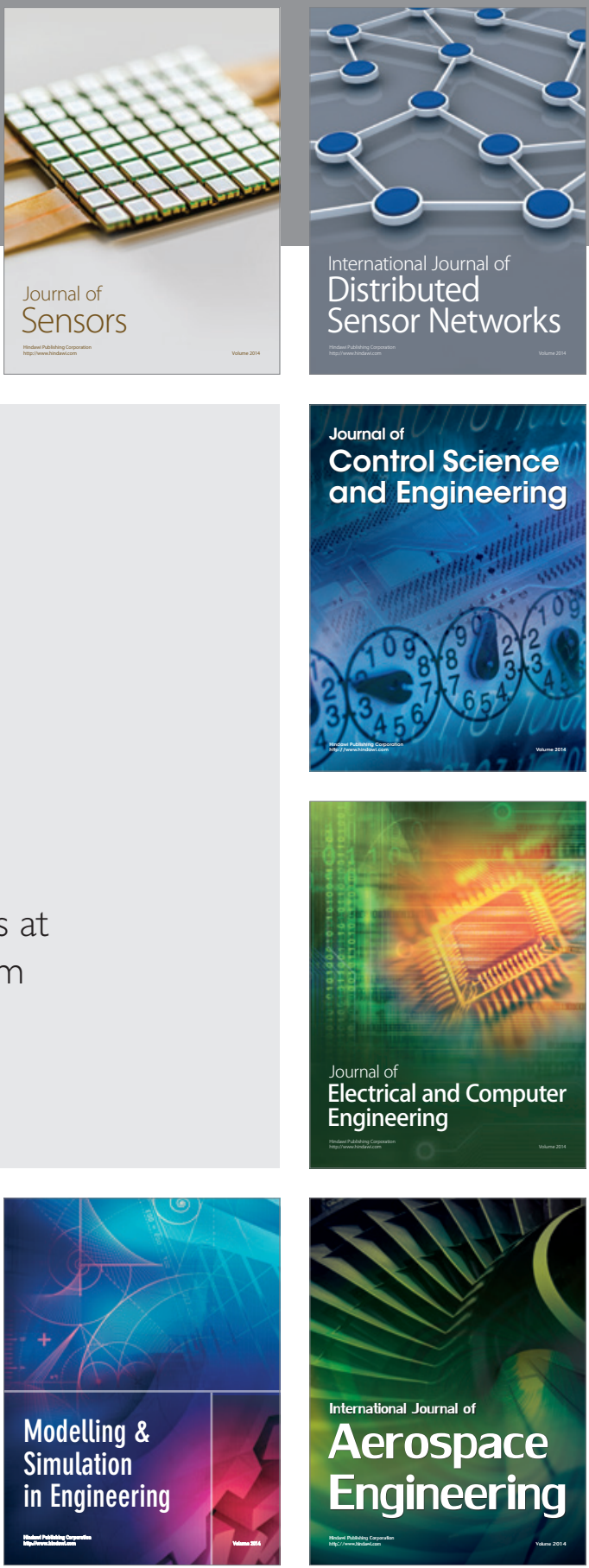

Journal of

Control Science

and Engineering
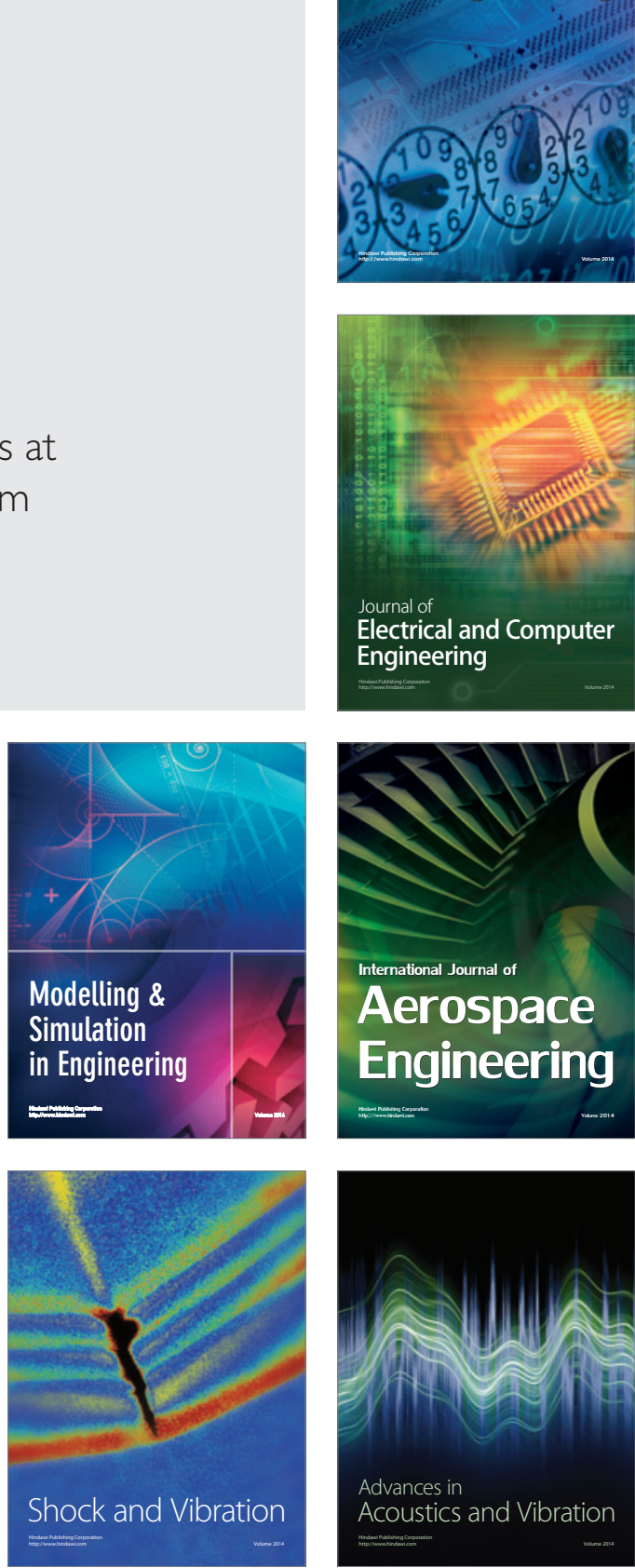